DESY $94-161$

ISSN $0418-9833$

hep-th/9410027

\title{
NEW EXAMPLES FOR WIGHTMAN FIELDS ON A MANIFOLD
}

\author{
M. KÖHLER $\dagger$ \\ II. INSTITUT FÜR THEORETISCHE PHYSIK, UNIVERSITÄT HAMBURG \\ LURUPER CHAUSSEE 149, D-22761 HAMBURG \\ GERMANY
}

PACS numbers: 1110, 0460, 0230

\begin{abstract}
The product of two free scalar fields on a manifold is shown to be a well defined operator valued distribution on the GNS Hilbert space of a globally Hadamard product state. Viewed as a new field all n-point distributions exist, giving a new example for a Wightman field on a manifold.
\end{abstract}

\section{INTRODUCTION}

Quantum field theory on curved spacetimes describes quantum fields propagating in a classical curved background. One of the main difficulties with such systems comes from the absence of Poincaré symmetry. On flat spacetime this symmetry fixes the vacuum states or equivalently allows a preferred class of representations of the canonical commutation relations (CCR) to be picked out. In the general case no analogous selection criterion exists. Since field theories have infinitely many degrees of freedom, different states may lead to unitarily inequivalent representations of the CCR. To deal with this fact the algebraic approach to quantum field theory by Haag and Kastler [8] seems appropriate. In this setting, roughly speaking, one describes the theory by a 'net of local algebras' which encodes the fields and observables. The positive linear functionals with unit norm on this net are called states. They describe the preparation of the system. However not all states are believed to be physical states. Nevertheless every state fixes -via the GNS construction- a Hilbert space, a 'vacuum vector' and a representation of the algebras, thus links the algebraic approach to the usual Hilbert space setting. Yet one might end up with unitarily inequivalent representations. Therefore it is not sufficient to construct the net; it is also necessary to characterize these physical states. One approach to this characterization are the 'scaling limit criterion' and the 'principle of local definiteness' introduced by Haag, Narnhofer and Stein [9] and further investigated by Fredenhagen and Haag in [5]. Both characterizations are designed for states on general quantum field theoretical models on arbitrary spacetimes. On the other hand, a class of states which is believed to be physical for the free, linear models are the quasifree Hadamard states: The singularity structure of their two-point distributions fulfils the 'Hadamard condition', e. g. it is fixed by the underlying geometry. (For a review see [11, 17] or the book of Fulling [6] and the references

Date: September 21, 1994.

$\dagger$ Supported by the DFG.

E-mail: mkoehler@x4u.desy.de. 
therein) However, besides from being a global condition, this condition has the disadvantage of being restricted to linear (free) models.

Radzikowski showed recently in [12 that the global Hadamard condition can equivalently be formulated locally in terms of wave front sets. Moreover, using wave front sets, he formulated a spectrum condition for quantum fields on a manifold, which might be useful as a new selection criterion for physical states. Unfortunately it can be shown that the n-point distributions of the free scalar field on a general spacetime violate his condition for $n>2$. We propose a slightly modified definition of a wave front set spectrum condition (WFSSC) for two-point distributions and give a nontrivial example of a Wightman field on a general spacetime satisfying this modified WFSSC. Starting from Hadamard states of two free, noninteracting Klein-Gordon fields, it is shown that their product is a new Wightman field on the spacetime together with a product state whose two-point distribution satisfies the new spectrum condition. Furthermore it will become clear that our method can be applied to other construction schemes initially based on Minkowski spacetime as well.

The organization of the work is as follows: After the Introduction we state the main result of this paper. For the convenience of the reader we continue by quoting some notions and results from the theory of pseudodifferential operators concerning distributions on manifolds; they are well known in the mathematical literature, but apparently were seldom used in physics. The notion of the wave front set of a distribution on a manifold is defined next and we relate this concept to Hadamard states. Our version of Radzikowski's spectrum condition follows. Finally the main Proposition is proved.

\section{The PRODUCT OF TWO FREE SCALAR FIELDS ON A CURVED SPACETIME}

We consider two free, noninteracting scalar fields $A$ and $B$ propagating on a spacetime which we assume to be a four dimensional, orientable, time orientable, globally hyperbolic Lorentz manifold with metric signature $(+,-,-,-)$. The classical Lagrangian of our model is given by

$$
\mathcal{L}=\frac{1}{2}\left(A_{; \mu} A^{; \mu}+\left(\xi R-m^{2}\right) A^{2}+B_{; \mu} B^{; \mu}+\left(\xi R-m^{2}\right) B^{2}\right) .
$$

$R$ is the scalar curvature and $\xi$ describes an additional coupling to the geometry. The equation of motion for the two fields obviously decouples giving the KleinGordon equation for both $A$ and $B$. We define a new field $K$ to be the pointwise product of the basic fields $A$ and $B$ respectively:

$$
K(x):=A(x) \cdot B(x)
$$

Consider two quasifree 'globally Hadamard' states ${ }^{A} \omega$ and ${ }^{B} \omega$ for the two scalar fields $A$ and $B$. Their tensor product $\omega \equiv{ }^{A} \omega \otimes{ }^{B} \omega$ defines a state for our model; it is called a globally Hadamard product state. The quantized fields $A$ and $B$ are mutually commuting operator valued distributions on the GNS Hilbertspace of $\omega$. Note that the r.h.s. of Eqn. (1) denotes the distributional product of $A$ and $B$ after quantization and such a product is not necessarily well defined a priori. The main result of this note is 
Proposition 1. $K$ is well defined and gives a new Wightman field on the GNS Hilbertspace $(H, \Omega, A, B)$ of a quasifree globally Hadamard product state $\omega$; the twopoint distribution of $K$ in this product state $\omega$ fulfils the wave front set spectrum condition (Definition 0 below).

For the proof of this proposition we need some properties of the wave front set of distributions, which are included in this work for the convenience of the reader. For further details and the proofs which are omitted, we refer the reader to the original literature ([10, 国) or to the monographs of Taylor and Reed \& Simon [14, 13].

The theory of wave front sets was developed in the seventies by Hörmander together with Duistermaat for their studies of pseudodifferential operators and differential equations on manifolds [10, [1. Wave front sets (WF) are refinements of the notion of the singular support (sing supp) of a distribution. One main reason for using them in favor of the sing supp is that they provide a simple characterization for the existence of products of distributions and eliminate the difference between local and global results. It is interesting that Duistermaat and Hörmander even mention relations between their 'microlocal analysis' which they used to study solutions of the Klein-Gordon equation on a manifold and quantum field theory. It seems however that their results did not find their way into the physical literature prior to the work of Radzikowski 12].

Definition 1. Let $v \in \mathcal{D}^{\prime}\left(\mathbb{R}^{n}\right)$ be a distribution on $\mathbb{R}^{n}$. The set $\Sigma_{z}(v)$ is the complement in $\mathbb{R}^{n} \backslash\{0\}$ of the set of all nonzero $\xi \in \mathbb{R}^{n}$ for which there is a smooth function $\phi$ with compact support not vanishing at $z$ and a conic neighborhood $C_{\xi}$ of $\xi$ such that for all $N \in \mathbb{Z}$ there exists a constant $C_{N}$ such that for all $\xi^{\prime} \in C_{\xi}$

$$
\left(1+\left|\xi^{\prime}\right|\right)^{N}\left|\widehat{\phi u}\left(\xi^{\prime}\right)\right| \leq C_{N}
$$

The hat ^ denotes Fourier transformation.

Definition 2. The wave front set of $v$ is defined by

$$
\mathrm{WF}(v)=\left\{(z, \theta) \in T^{*} \mathbb{R}^{n} \backslash\{0\} \mid \quad \theta \in \Sigma_{z}(v)\right\}
$$

All points $\left\langle x, \xi>\in T^{*} \mathbb{R}^{n}\right.$ which are not in the wave front set are called regular directed points.

Remark . 1. $\operatorname{WF}(v)$ is a closed subset of $T^{*} \mathbb{R}^{n} \backslash\{0\}$ since each regular directed point $<x, \xi>\notin \mathrm{WF}(v)$ has by definition an open neighborhood in $T^{*} \mathbb{R}^{n} \backslash\{0\}$ consisting of regular directed points, too.

2. The wave front set of a smooth function is the empty set

3. For $v \in \mathcal{D}^{\prime}\left(\mathbb{R}^{n}\right)$ with wave front set $\mathrm{WF}(v)$, the projection of the wave front set to the base point gives the singular support of $v$.

4. For all smooth functions $\tilde{\phi}$ with compact support $\mathrm{WF}(\tilde{\phi} v) \subset \mathrm{WF}(v)$.

5. For two distributions $v, w \in \mathcal{D}^{\prime}\left(\mathbb{R}^{n}\right)$ with wave front sets $\operatorname{WF}(v)$ and $\operatorname{WF}(w)$ respectively, the wave front set of $(v+w) \in \mathcal{D}^{\prime}\left(\mathbb{R}^{n}\right)$ is contained in $\operatorname{WF}(v) \cup$ $\mathrm{WF}(w)$

In order to extend this definition to manifolds we use the behavior of $\operatorname{WF}(v)$ under diffeomorphisms of $\mathbb{R}^{n}$ to $\mathbb{R}^{n}$ (Theorem 1 below). Although Definition 1 contains a Fourier transform, it turns out that the elements of $\mathrm{WF}(v)$ transform as elements of $T^{*} \mathbb{R}^{n}$, the cotangential bundle of $\mathbb{R}^{n}$. It is therefore possible to define the wave front set of a distribution on a manifold via charts. 
Theorem 1 (Theorem IX.44 and Problem 75 in $\sqrt{13}$ ). Let $v \in \mathcal{D}^{\prime}\left(\mathbb{R}^{n}\right)$ be a distribution on $\mathbb{R}^{n}$, $\chi$ be a diffeomorphism of $\mathbb{R}^{n}$ to $\mathbb{R}^{n}$ and let $v \circ \chi$ be the distribution

$$
v \circ \chi(f):=v\left(g^{-1}\left(f \circ \chi^{-1}\right)\right)
$$

where $g$ is the determinant of the Jacobian matrix $d \chi$. Define $\chi_{*}: \mathbb{R}^{n} \times\left(\mathbb{R}^{n} \backslash\{0\}\right) \rightarrow$ $\mathbb{R}^{n} \times\left(\mathbb{R}^{n} \backslash\{0\}\right)$ by

$$
\chi_{*}<x, \xi>=<\chi(x), d \chi^{*}(\xi)>
$$

where $d \chi^{*}$ is the adjoint of $d \chi$ with respect to the Euclidean inner product on $\mathbb{R}^{n}$. Then

$$
\mathrm{WF}(v \circ \chi)=\chi_{*}(\mathrm{WF}(v))
$$

Definition 3. Let $u \in \mathcal{D}^{\prime}(M)$ be a distribution over some manifold $M$. Let $\left\{X_{\lambda}\right\}_{\lambda \in \mathbb{N}}$ denote an open covering of $M$. Choose a compatible partition of unity $\left\{\Phi_{\lambda}\right\}_{\lambda \in \mathbb{N}}$. Without loss of generality we may assume that $\operatorname{supp} \Phi_{\lambda}$ is contained in a single coordinate patch for every $\lambda \in \mathbb{N}$. The corresponding charts are denoted by $\chi_{\lambda}: \operatorname{supp} \Phi_{\lambda} \subset U \rightarrow \mathbb{R}^{n}$. Using the same notation as in Theorem $\mathbb{1}$ above we find as a Corollary

$$
\chi_{\lambda *}^{-1}\left(\mathrm{WF}\left(\Phi_{\lambda} u\right)\right) \equiv \operatorname{WF}\left(\Phi_{\lambda} u \circ \chi_{\lambda}^{-1}\right)
$$

where $\Phi_{\lambda} u \circ \chi_{\lambda}^{-1}$ obviously is a distribution with compact support over $\mathbb{R}^{n}$. We define the wave front set of $u$ by

$$
\mathrm{WF}(u)=\bigcup_{\lambda} \mathrm{WF}\left(\Phi_{\lambda} u\right)
$$

Remark. This is a local definition. It follows that the following results which are stated in 13] for distributions on $\mathbb{R}^{n}$, are valid in the generic case, too.

A useful application of wave front sets is the definition of products of distributions. Wave front sets provide a simple characterization for the existence of such products, which furthermore can be extended to manifolds. The following definition of a product and its relation to wave front sets can be found for example in Reed \& Simon [13, p.90-97].

Definition 4. Let $v, w \in \mathcal{D}^{\prime}\left(\mathbb{R}^{n}\right)$. The distribution $T \in \mathcal{D}^{\prime}\left(\mathbb{R}^{n}\right)$ is the product of $v$ and $w$ if and only if for all $x \in \mathbb{R}^{n}$ there exists a smooth function $f$ not vanishing at $x$ such that for all $k \in \mathbb{R}^{n}$

$$
\widehat{f^{2} T}(k)=(2 \pi)^{-n / 2} \int_{\mathbb{R}^{n}} \widehat{f v}(l) \widehat{f w}(k-l) d^{n} l
$$

where the integral is absolutely convergent.

Remark . - The product is well defined, since if such a $T$ exists, it is unique:

Let $g \in \mathcal{D}\left(\mathbb{R}^{n}\right)$ then

$$
\widehat{g f^{2} T}=(2 \pi)^{-n / 2} \widehat{g f v} * \widehat{f w}=(2 \pi)^{-n / 2} \widehat{f v} * \widehat{g f w},
$$

since the change of variables is legitimate due to the assumption of absolute convergence of (4). Now suppose $T_{1}$ and $T_{2}$ both fulfil (4), e.g. for all $x \in \mathbb{R}^{n}$ there exist functions $f$ and $g$ not vanishing at $x$ such that $\widehat{f^{2} T_{1}}=\widehat{f v} * \widehat{f w}$ and $\widehat{g^{2} T_{2}}=\widehat{g v} * \widehat{g w}$. We conclude $\widehat{f^{2} g^{2} T_{1}}=\widehat{f^{2} g^{2} T_{2}}$, so $\left(T_{1}-T_{2}\right)$ vanishes near $x$ for all $x$, that is it is zero. 
- The product of two distributions with disjoint support is zero.

- Since this is a local definition, we can extend it immediately to manifolds, using charts (See 10]).

The following Theorem gives the relation between the wave front sets of two distributions and the existence of their product.

Theorem 2 (Theorem IX.54 of [13]). Let $v, w$ be two distributions on $M$ such that

$$
\mathrm{WF}(v) \oplus \mathrm{WF}(w) \equiv\left\{<x, k_{1}+k_{2}>\mid \quad<x, k_{1}>\in \mathrm{WF}(v),<x, k_{2}>\in \mathrm{WF}(w)\right\}
$$

does not contain any element of the form $\langle x, 0\rangle$, then the product $v \cdot w$ exists and has wave front set

$$
\mathrm{WF}(v \cdot w) \subset \mathrm{WF}(v) \cup \mathrm{WF}(w) \cup(\mathrm{WF}(v) \oplus \mathrm{WF}(w))
$$

\section{HADAMARD STATES}

The Hadamard condition for quasifree states of scalar fields on a manifold is believed to be a necessary condition for physical states since the work of DeWitt and Brehme in 1960 [3] and was intensively studied since this time by various authors (See the references in Fulling's book [6]). However only recently Kay and Wald 11] gave a mathematically rigorous definition of this condition.

In this section we recall their precise characterization of the global Hadamard condition. We continue by calculating the wave front set of the corresponding states using the 'Propagation of Singularities Theorem' of Duistermaat and Hörmander (Theorem 6.1 .1 of 田). The reverse relation, e.g. a characterization of globally Hadamard states by their wave front sets due to Radzikowski [12], is quoted next; it is followed by our version of his wave front set spectrum condition.

Let $\omega_{2}$ be the two-point distribution of a quasifree state on the Borchers Uhlmann algebra 11, 15 for a scalar field on a globally hyperbolic manifold $\left(M, g_{a b}\right)$ satisfying the Klein-Gordon equation and local commutativity. Assume that a preferred time orientation has been chosen on $\left(M, g_{a b}\right)$ and let $T$ be a global time function increasing towards the future. Recall the definition of a convex normal neighborhood: An open subset $U \subset M$ is called a convex normal neighborhood if for all points $x_{1}, x_{2} \in U$ there exists a unique geodesic contained in $U$ connecting $x_{1}$ and $x_{2}$. Let $\mathcal{O} \subset M \times M$ be an open neighborhood in $M \times M$, of the set of causally related points $\left(x_{1}, x_{2}\right)$ such that $J^{+}\left(x_{1}\right) \cap J^{-}\left(x_{2}\right)$ and $J^{+}\left(x_{2}\right) \cap J^{-}\left(x_{1}\right)$ are contained within a convex normal neighborhood. As usual $J^{ \pm}(x)$ denotes the causal future (past) of the point $x$. The square of the geodesic distance, $\sigma$, is well defined and smooth on $\mathcal{O}$. For each integer $p$ and $\epsilon \geq 0$ define for $\left(x, x^{\prime}\right) \in \mathcal{O}$ the complex valued function

$$
\begin{aligned}
& G_{\epsilon}^{T, p}\left(x, x^{\prime}\right) \\
& \quad=\frac{1}{(2 \pi)^{2}}\left(\frac{\Delta^{1 / 2}\left(x, x^{\prime}\right)}{\sigma\left(x, x^{\prime}\right)+2 i \epsilon t+\epsilon^{2}}+v^{(p)}\left(x, x^{\prime}\right) \ln \left[\sigma\left(x, x^{\prime}\right)+2 i \epsilon t+\epsilon^{2}\right]\right)
\end{aligned}
$$

where $t \equiv T(x)-T\left(x^{\prime}\right)$ and $\Delta^{1 / 2}$ and $v^{(p)}$ are smooth functions uniquely determined by the geometry on $M\left(\Delta^{1 / 2}\right.$ is the van Vleck Morette determinant and $v^{(p)}$ is given by the Hadamard recursion relation up to order $p$ [3, 2]. The branch cut of the logarithm is taken to lie on the negative real axis). Let $\mathcal{C}$ be a Cauchy surface and let $N$ be a neighborhood of $\mathcal{C}$ with the property: For each pair of points $x_{1}, x_{2} \in N$ such that $x_{1}$ can be reached by a causal curve emerging from $x_{2}\left(x_{1} \in J^{+}\left(x_{2}\right)\right)$ one 
can find a convex normal neighborhood in $M$ containing $J^{-}\left(x_{1}\right) \cap J^{+}\left(x_{2}\right) . \quad N$ is called a causal normal neighborhood of $\mathcal{C}$. Now let $\mathcal{O}^{\prime}$ be a neighborhood in $N \times N$ such that its closure is contained in $\mathcal{O}$ and choose a smooth real valued function $\chi$ on $M \times M$ with the properties $\chi(x, y)=0$ if $(x, y) \notin \mathcal{O}$ and $\chi(x, y)=1$ if $(x, y) \in \mathcal{O}^{\prime}$ respectively.

Definition 5 (Globally Hadamard states). We say the state mentioned above is globally Hadamard iff its two-point distribution $\omega_{2}$ is such that for each integer $p$ there exists a $C^{p}$ - function $H^{(p)}(x, y)$ on $N \times N$ such that for all $F_{1}, F_{2} \in C_{0}^{\infty}(N)$ we have

$$
\begin{aligned}
\omega_{2}\left(F_{1}\right. & \left.\otimes F_{2}\right) \\
& =\lim _{\epsilon \rightarrow 0}\left(\int_{N \times N}\left(\chi(x, y) G_{\epsilon}^{T, p}(x, y)+H^{(p)}(x, y)\right) F_{1}(x) F_{2}(y) d \mu_{x} d \mu_{y}\right)
\end{aligned}
$$

For a detailed discussion of this definition the reader is referred to the work of Kay and Wald [11.

Remark. Kay and Wald prove in [11 that this Definition is independent of the Cauchy surface $\mathcal{C}$. This in turn means that the Cauchy evolution preserves the Hadamard structure, e. g. $\omega_{2}$ restricted to a causal normal neighborhood $N$ of a Cauchy surface $\mathcal{C}$ already fixes $\omega_{2}$ throughout the whole spacetime.

3.1. The wave front set of a globally Hadamard state. The result of this subsection (Corollary 1 below) can already be found in the dissertation of Radzikowski. However the proof he gave is -as it stands- only valid for the rather trivial case of a flat spacetime. The proof presented here verifies his claim in the general case.

Following Radzikowski's line of argument, consider first the free massive KleinGordon field on Minkowski space in the vacuum state. The vacuum is known to fulfil the global Hadamard condition with $\Delta^{1 / 2} \equiv 1$ and $v^{(p)} \equiv \sum_{n=0}^{p} 2\left(\frac{m^{2}}{2}\right)^{n+1} / n !(n+$ 1)! ([2]). The wave front set of the corresponding two-point distribution ${ }^{\mathrm{Mk}} \omega_{2}^{m}$ is also well known (See for instance [13]):

Theorem 3 (Theorem IX.48 of [13]). The two-point distribution ${ }^{\mathrm{Mk}} \omega_{2}^{m}$ of the free massive scalar field on Minkowski space in the vacuum state has wave front set

$$
\begin{aligned}
& \operatorname{WF}\left({ }^{\mathrm{Mk}} \omega_{2}^{m}\right) \\
& \begin{aligned}
\left\{\left(x_{1}, k_{1}\right),\left(x_{2}, k_{2}\right) \in \mathbb{R}^{4} \times\left(\mathbb{R}^{4} \backslash\{0\}\right) \mid\right. & x_{1} \neq x_{2} ;\left(x_{1}-x_{2}\right)^{2}=0 ; \\
& k_{1} \|\left(x_{1}-x_{2}\right) ; k_{1}+k_{2}=0 ; \\
& \left.k_{1}^{0} \geq 0\right\}
\end{aligned} \\
& \bigcup\left\{\left(x, k_{1}\right),\left(x, k_{2}\right) \in \mathbb{R}^{4} \times\left(\mathbb{R}^{4} \backslash\{0\}\right) \mid \quad k_{1}+k_{2}=0 ; k_{1}^{2}=0 ; k_{1}^{0} \geq 0\right\}
\end{aligned}
$$

For a proof see 13] or use the following representation of the Fourier transform of ${ }^{\mathrm{Mk}} \omega_{2}^{m}$ :

$$
\widehat{\mathrm{Mk}} \omega_{2}^{m}=(2 \pi)^{-1} \delta\left(k_{1}+k_{2}\right) \Theta\left(k_{1}^{0}\right) \delta\left(k_{1}^{2}-m^{2}\right) \in \mathcal{S}^{\prime}\left(\mathbb{R}^{4} \times \mathbb{R}^{4}\right)
$$

Using this representation and Definition 2 it is easy to prove the Theorem explicitly.

In order to extend this result to arbitrary manifolds, let $x \in M$ be a point on a Cauchy surface $\mathcal{C}$ and let $U_{x}$ be a convex normal neighborhood of $x$. Denote by $\mathcal{C}_{b} \subset \mathcal{C}$ an arbitrary (non void) open subset of $\mathcal{C}$ such that the domain of causal 
dependence, $D\left(\mathcal{C}_{b}\right)$, is contained in $U_{x}$. We are going to calculate the wavefront set of $\omega_{2}$ for all base points $x_{1}, x_{2} \in D^{+}\left(\mathcal{C}_{b}\right):=D\left(\mathcal{C}_{b}\right) \cap J^{+}\left(\mathcal{C}_{b}\right)$ using some properties of Hadamard states on a special smooth deformation $\left(\hat{M}, \hat{g}_{a b}\right)$ of our original spacetime. Finally the Propagation of Singularities Theorem (Theorem 1 below) and our knowledge that $\omega_{2}$ has no singularities for space-like separated points allows us to extend this result to the whole spacetime $\left(M, g_{a b}\right)$.

Given $\mathcal{C}, x \in \mathcal{C}$ and $\mathcal{C}_{b}$ it will be shown below that there exists a globally hyperbolic spacetime $\left(\tilde{M}, \tilde{g}_{a b}\right)$ with the following properties:

(i) A neighborhood $U$ of $\mathcal{C}$ in $M$ is isometrically isomorphic to a neighborhood $\tilde{U}$ in $\tilde{M}$ and the isometry $\phi$ is also an isometry between $\mathcal{C}$ and $\tilde{\mathcal{C}}$; e. g. $\left(\tilde{M}, \tilde{g}_{a b}\right)$ is a smooth deformation of $\left(M, g_{a b}\right)$.

(ii) For all points $x_{1}, x_{2} \in D^{+}\left(\mathcal{C}_{b}\right)$ there exists a Cauchy surface $\tilde{S}$ with neighborhood $\hat{U}$ in $\tilde{M}$, such that the metric $\tilde{g}_{a b}$ restricted to $\hat{U}$ is flat (i.e. Minkowskian) and $D\left(\phi\left(\mathcal{C}_{b}\right)\right) \subset D(\hat{U})$.

Now $\omega_{2}$ induces canonically a Hadamard distribution $\tilde{\omega}_{2}$ on $\left(\tilde{M}, \tilde{g}_{a b}\right)$, since it does so on $\tilde{U}$ : Being a Hadamard distribution on $\tilde{U}$ implies, by the Remark following Definition 5, that $\tilde{\omega}_{2}$ is a Hadamard distribution throughout $\left(\tilde{M}, \tilde{g}_{a b}\right)$. Furthermore, the wave front set of $\tilde{\omega}_{2}$ on $\tilde{U}$ determines that of $\omega$ at $U$ and vice versa by Theorem 1. On the other hand, using the Propagation of Singularities Theorem and the smoothness of $\tilde{\omega}_{2}$ for space-like separated points, the wave front set of $\tilde{\omega}_{2}$ at $\tilde{x}_{1}=\phi\left(x_{1}\right)$ and $\tilde{x}_{2}=\phi\left(x_{2}\right)$ is already fixed by the wave front set of that distribution at all points in $\hat{U}$ (See below and note that $\tilde{x}_{1}$ and $\tilde{x}_{2} \in D(\hat{U})$ ). To obtain the wave front set of $\omega_{2}$ at $x_{1}$ and $x_{2}$ it is therefore sufficient to calculate the wave front set of $\tilde{\omega}_{2}$ for all points in $\hat{U}$.

The following computations are performed entirely in the flat part of $\tilde{M}$ and were partly sketched in 12]. To keep the notation simple we omit the ${ }^{\sim}$ in what follows. Note first that Eqn. (6) is valid in $\left(\tilde{M}, \tilde{g}_{a b}\right)$, too. Choose a point $x \in S \equiv \hat{\mathcal{C}}\left(t_{1}\right)$ and a convex normal neighborhood $U_{x} \subset \hat{U}$ of $x$. Using normal coordinates and an adapted time function $T$ the distribution $G_{\epsilon}^{T, p}$, when restricted to $U_{x} \times U_{x}$ is

$$
\begin{aligned}
& G^{p}:= \\
& \lim _{\epsilon \rightarrow 0} \chi G_{\epsilon}^{p}=\lim _{\epsilon \rightarrow 0} \frac{\Delta^{1 / 2}\left(x_{1}, x_{2}\right)}{-\left(x_{1}-x_{2}\right)^{2}+2 i \epsilon\left(x_{1}^{0}-x_{2}^{0}\right)+\epsilon^{2}} \\
& +v^{(p)}\left(x_{1}, x_{2}\right) \ln \left(-\left(x_{1}-x_{2}\right)^{2}+2 i \epsilon\left(x_{1}^{0}-x_{2}^{0}\right)+\epsilon^{2}\right)
\end{aligned}
$$

The metric $g_{a b}$ is flat on $U_{x}$ and hence $2 \sigma(x, y) \equiv-\left(x_{1}-x_{2}\right)^{2}$ in these coordinates. Furthermore the Van Vleck Morette determinant $\Delta^{1 / 2}$ and $v^{(p)}$ are identical to the corresponding functions on Minkowski space, since only local properties of the underlying geometry enter into the Hadamard recursion relations. We conclude that $\mathrm{WF}\left({ }^{\mathrm{Mk}} \omega_{2}^{0}\right)=\mathrm{WF}\left(G^{p}\right)$ in normal coordinates on $U_{x}^{\prime} \times U_{x}^{\prime}$. Using Definition 3 this result can be pulled back to the manifold:

$$
\begin{aligned}
& \mathrm{WF}\left(G^{p}\right)=\left\{\left(x_{1}, k_{1}\right),\left(x_{2}, k_{2}\right) \in\left(T^{*} M \times T^{*} M\right) \backslash\{0\} \mid\right. \\
&\left(x_{1}, k_{1}\right)\left.\sim\left(x_{2},-k_{2}\right) ; \quad k_{1}^{0} \geq 0\right\} \quad \text { on } U_{x}^{\prime} \times U_{x}^{\prime}
\end{aligned}
$$

\footnotetext{
${ }^{1}$ The author thanks R. Verch for calling his attention to the deformation argument of Fulling, Narcowich and Wald [7]. Note that the metric $g_{a b}$ restricted to $D^{+}\left(\mathcal{C}_{b}\right)$ is not flat in general; the latter was assumed implicitly in Radzikowski's argument at some point, making the following modification of his proof necessary.
} 
where $\left(x_{1}, k_{1}\right) \sim\left(x_{2}, k_{2}\right)$ means (i) $x_{1}$ and $x_{2}$ can be joined by a null geodesic $\gamma$, (ii) $k_{1}\left(=k_{1 \nu}\right)$ is a cotangent vector such that $k_{1}^{\mu}=k_{1 \nu} g^{\mu \nu}$ is tangential to $\gamma$, (iii) The parallel transport of $k_{1}$ along $\gamma$ yields $k_{2}$ or (i) $x_{1}=x_{2}$, (ii) $k_{1}^{2}=0$ and (iii) $k_{1}=k_{2}$. Now $H^{(p)} \in C^{p}(M \times M)$ which implies for $\phi \in C_{0}^{\infty}(M)$ and $\phi(x) \neq 0$

$$
\left((\phi \otimes \phi) H^{(p)} \widehat{)}\left(k_{1}, k_{2}\right) \leq C_{p}(1+|k|)^{-p}\right.
$$

for a constant $C_{p}$ and all $k=\left(k_{1}, k_{2}\right)$. Since $\omega_{2}=G^{p}+H^{(p)}$ for all $p$ we conclude that the wave front set of $\omega_{2}$ restricted to $U_{x}^{\prime} \times U_{x}^{\prime}$ is

$$
\begin{aligned}
\mathrm{WF}\left(\omega_{2}\right)=\left\{\left(x_{1}, k_{1}\right),\left(x_{2}, k_{2}\right) \in\right. & \left(T^{*} M \times T^{*} M\right) \backslash\{0\} \mid \\
\left(x_{1}, k_{1}\right) & \left.\sim\left(x_{2},-k_{2}\right) ; \quad k_{1}^{0} \geq 0\right\} \quad \text { on } U_{x}^{\prime} \times U_{x}^{\prime}
\end{aligned}
$$

As a Corollary to the following Theorem of Hörmander this extends to all of $\tilde{M}$.

Theorem 4 (Corollary to Theorem 6.1 .1 of 国). Let $P=\mathbf{1} \otimes\left(\square+\nabla_{\mu} V^{\mu}+b\right)$ be a pseudodifferential operator on a globally hyperbolic manifold $\left(M, g_{a b}\right)$. $\square$ denotes the D'Alembert operator and $V^{\mu}$ and $b$ are a smooth vector field and smooth function on $M$ respectively. The principal symbol of $P$ is denoted by $p$ and is given here by

$$
\begin{aligned}
p:\left(T^{*} M \times T^{*} M\right) \backslash\{0\} & \rightarrow \mathbb{R} \\
\left(x_{1}, k_{1} ; x_{2}, k_{2}\right) & \mapsto g^{\mu \nu}\left(x_{2}\right) k_{2 \mu} k_{2 \nu}
\end{aligned}
$$

If $u \in \mathcal{D}^{\prime}(M \times M)$ is a weak solution of $P u=0$ with wave front set $\mathrm{WF}(u)$, it follows that

a) $\mathrm{WF}(u) \subseteq p^{-1}(0)$ and

b) $\mathrm{WF}(u)$ is invariant under the (Hamiltonian) vector field $H_{p}$ given by

$$
H_{p}=\sum_{i=1}^{2 n} \frac{\partial p(x, k)}{\partial x_{i}} \frac{\partial}{\partial k_{i}}-\frac{\partial p(x, k)}{\partial k_{i}} \frac{\partial}{\partial x_{i}}
$$

in local coordinates.

Definition 6. The bicharacteristic strips of $P$ are the curves on the submanifold $p^{-1}(0) \subset\left(T^{*} M \times T^{*} M\right) \backslash\{0\}$ which are generated by $H_{p}$.

Note that b) means: If $\left(x_{1}, k_{1} ; x_{2}, k_{2}\right)$ and $\left(x_{1}^{\prime}, k_{1}^{\prime} ; x_{2}^{\prime}, k_{2}^{\prime}\right)$ are on the same bicharacteristic strip, denoted by $\left(x_{1}, k_{1} ; x_{2}, k_{2}\right) \approx\left(x_{1}^{\prime}, k_{1}^{\prime} ; x_{2}^{\prime}, k_{2}^{\prime}\right)$ and $\left(x_{1}, k_{1}, x_{2}, k_{2}\right) \in$ $\mathrm{WF}(u)$, then $\left(x_{1}^{\prime}, k_{1}^{\prime} ; x_{2}^{\prime}, k_{2}^{\prime}\right) \in \mathrm{WF}(u)$. For $P=\mathbf{1} \otimes\left(\square+\nabla_{\mu} V^{\mu}+b\right)$ one finds (See Proposition 2.8 in [12])

$$
\left(x_{1}, k_{1} ; x_{2}, k_{2}\right) \approx\left(x_{1}^{\prime}, k_{1}^{\prime} ; x_{2}^{\prime}, k_{2}^{\prime}\right) \Leftrightarrow\left(x_{1}, k_{1}\right)=\left(x_{1}^{\prime}, k_{1}^{\prime}\right) \quad \text { and } \quad\left(x_{2}, k_{2}\right) \sim\left(x_{2}^{\prime}, k_{2}^{\prime}\right)
$$

Corollary 1 (See Theorem 2.6 in [12]). The two-point distribution $\omega_{2}$ of a free massive Klein-Gordon field on a globally hyperbolic spacetime in a globally Hadamard state has wave front set

$$
\begin{aligned}
& \mathrm{WF}\left(\omega_{2}\right)=\left\{\left(x_{1}, k_{1}\right),\left(x_{2},-k_{2}\right) \in\left(T^{*} M \times T^{*} M\right) \backslash\{0\} \mid\right. \\
&\left.\left(x_{1}, k_{1}\right) \sim\left(x_{2}, k_{2}\right) ; \quad k_{1}^{0} \geq 0\right\}
\end{aligned}
$$

Proof. Since $\omega_{2}$ is a bisolution of the Klein-Gordon operator, we can apply Theorem 0 with $P_{1}=\mathbf{1} \otimes\left(\square+m^{2}\right)$ or $P_{2}=\left(\square+m^{2}\right) \otimes \mathbf{1}$. In the first case we conclude by a) that

$$
\mathrm{WF}\left(\omega_{2}\right) \subseteq\left\{\left(x_{1}, k_{1} ; x_{2}, k_{2}\right) \in\left(T^{*} M \times T^{*} M\right) \backslash\{0\} \mid \quad k_{2}^{2}=0\right\}
$$


and -using b)-

$$
\left(x_{1}, k_{1} ; x_{2}, k_{2}\right) \in \mathrm{WF}\left(\omega_{2}\right) \wedge\left(x_{2}, k_{2}\right) \sim\left(x_{2}^{\prime}, k_{2}^{\prime}\right) \Rightarrow\left(x_{1}, k_{1} ; x_{2}^{\prime}, k_{2}^{\prime}\right) \in \mathrm{WF}\left(\omega_{2}\right)
$$

Obviously the second case leads to

$$
\mathrm{WF}\left(\omega_{2}\right) \subseteq\left\{\left(x_{1}, k_{1} ; x_{2}, k_{2}\right) \in\left(T^{*} M \times T^{*} M\right) \backslash\{0\} \mid \quad k_{1}^{2}=0\right\}
$$

and

$$
\begin{aligned}
& \left(x_{1}, k_{1} ; x_{2}, k_{2}\right) \in \mathrm{WF}\left(\omega_{2}\right) \wedge\left(x_{1}, k_{1}\right) \sim\left(x_{1}^{\prime}, k_{1}^{\prime}\right) \\
& \Rightarrow\left(x_{1}^{\prime}, k_{1}^{\prime} ; x_{2}, k_{2}\right) \in \mathrm{WF}\left(\omega_{2}\right)
\end{aligned}
$$

To decide whether $\left(x_{1}, k_{1} ; x_{2}, k_{2}\right)$ with $k_{1}^{2}=k_{2}^{2}=0$ is in the wave front set of $\omega_{2}$, one checks first whether $x_{1}$ and $x_{2}$ are space-like separated. If this is true, then $\left(x_{1}, k_{1} ; x_{2}, k_{2}\right) \notin \mathrm{WF}\left(\omega_{2}\right)$, since the projection of the wave front set of a distribution to the base point gives the singular support of that distribution; but $\omega_{2}$ is assumed to have no singularities at space-like separated points. If they are causally related, we use Eqn. (10) and (11) to propagate $\left(x_{i}, k_{i}\right)$ along the null geodesic $\gamma_{i}$ with tangent vector $k_{i}$ at $x_{i}$ to the Cauchy surface $\mathcal{C}$. Note that due to the global hyperbolicity of $M$ this is always possible. If the points on $\mathcal{C}$ do not coincide, we have $\left(x_{1}, k_{1} ; x_{2}, k_{2}\right) \notin \mathrm{WF}\left(\omega_{2}\right)$, since different points on a Cauchy surface are spacelike separated. Should they coincide at say $x \in \mathcal{C}$, we check whether this particular combination $\left(x, k_{1} ; x, k_{2}\right)$ is in the wave front set using Eqn. (8).

To finish the argument, we have to construct a spacetime $\left(\tilde{M}, \tilde{g}_{a b}\right)$ that satisfies the properties (i) and (ii). This can be done by using the methods of Appendix $\mathrm{C}$ in [7] (See also [16]). Let $D\left(\mathcal{C}_{b}\right) \subset V \subset N$ be a causal normal neighborhood of $\mathcal{C}$, such that $\mathcal{C}$ is also a Cauchy surface for $V . V$ is, by the normal exponential map $\phi$ of $\mathcal{C}$, diffeomorphic to an open neighborhood $\tilde{V} \subset \mathbb{R} \times \mathcal{C}$ of $\tilde{\mathcal{C}}=\{0\} \times \mathcal{C}=\phi(\mathcal{C})$. Using normal coordinates $(t, \mathbf{x})$ around some point in $\tilde{\mathcal{C}}$, the metric $\phi_{*} g$ on $\tilde{V}$ takes the form

$$
d t^{2}-h_{i j}(t, \mathbf{x}) d \mathrm{x}^{i} d \mathrm{x}^{j}
$$

Choose $t_{1}<t_{2}<t_{3}<0$ such that

$$
\overline{\hat{\mathcal{C}}\left(t_{i}\right) \cap J^{-}\left(\tilde{\mathcal{C}_{b}}\right)} \subset \phi\left(U_{x}\right) \quad i=1,2,3,
$$

where $\hat{\mathcal{C}}(t):=\{t\} \times \mathcal{C}, t \in \mathbb{R}$ and $\tilde{\mathcal{C}}_{b}:=\phi\left(\mathcal{C}_{b}\right)$ (As a consequence of the global hyperbolicity and due to the assumption $D\left(\mathcal{C}_{b}\right) \subset U_{x}$ such a choice is always possible). Recall that $\phi\left(U_{x}\right)$ can be covered with a single coordinate patch by assumption. Next choose a neighborhood $\tilde{U}$ of $\tilde{\mathcal{C}}$ such that $\overline{\tilde{U}} \subset \tilde{V} \cap \operatorname{int}\left(J^{+}\left(\hat{\mathcal{C}}\left(t_{3}\right)\right)\right)$. Due to the fact that $t_{1}<t_{2}$ there exists a neighborhood $\hat{U}$ of $\overline{J^{-}\left(\tilde{\mathcal{C}_{b}}\right) \cap \hat{\mathcal{C}}\left(t_{1}\right)}$ in $\tilde{M}$ such that (i) $\overline{\hat{U}} \subset \phi\left(U_{x}\right)$ and (ii) $\overline{\hat{U}} \cap J^{+}\left(\hat{\mathcal{C}}\left(t_{2}\right)\right)=\emptyset$. Let $f \in C^{\infty}(\mathbb{R} \times \mathcal{C}, \mathbb{R})$ be a smooth function $0 \leq f \leq 1, f \equiv 0$ on $\tilde{U}$ and $f \equiv 1$ outside the closure of $\tilde{V}$ or in the past of $J^{-}\left(\hat{\mathcal{C}}\left(t_{2}\right)\right)$. Let $\tilde{h}$ be a complete Riemannian metric on $\mathcal{C}$ being flat on the spatial component of $\hat{U} \cap\left(\hat{\mathcal{C}}\left(t_{3}\right)\right)$. Note that the existence of such a $\tilde{h}$ follows from the fact that $\overline{\hat{U}}$ can be covered by a single coordinate patch (i.e. $\left.\phi\left(U_{x}\right)\right)$. Let $\beta \in C^{\infty}\left(\mathbb{R} \times \mathcal{C}, \mathbb{R}^{+}\right)$be a function equal to one on $\tilde{U}$ and on $\left(-\infty, t_{2}\right)$. Define a Lorentzian metric $\tilde{g}_{a b}$ on $\mathbb{R} \times \mathcal{C}$ by setting in the coordinates above the coordinate expression of $\tilde{g}_{a b}$ equal to

$$
\beta(t, \mathbf{x}) d t^{2}-\left((1-f(t, \mathbf{x})) h_{i j}(t, \mathbf{x})+f(t, \mathbf{x}) \tilde{h}_{i j}(t, \mathbf{x})\right) d \mathbf{x}^{i} d \mathbf{x}^{j}
$$


By choosing $\beta$ sufficiently small outside the region where it is demanded to be on ${ }^{2}$, we can ensure that $\left(\tilde{M}:=\mathbb{R} \times \mathcal{C}, \tilde{g}_{a b}\right)$ is globally hyperbolic. Setting $U:=\phi^{-1}(U)$ and $\tilde{S}:=\phi^{-1}\left(\hat{\mathcal{C}}\left(t_{1}\right)\right)$ finishes the construction.

A first consequence of Corollary 1 is

Corollary 2. Let $\omega_{n}$ be the n-point distribution arising from a quasifree Hadamard state of a massive Klein-Gordon field propagating on a globally hyperbolic spacetime $\left(M, g_{a b}\right)$. Then all finite powers of $\omega_{n}$ exist as products of distributions.

Proof. Assuming all odd n-point distributions to vanish, we have since $\omega_{n}$ arises from a quasifree state

$$
\omega_{n}\left(x_{1}, \ldots, x_{n}\right)=\sum_{P} \prod_{r} \omega_{2}\left(x_{(r, 1)}, x_{(r, 2)}\right)
$$

where $P$ denotes a partition of the set of points $\left\{x_{i}\right\}$ into subsets which are pairings of points, labeled by $r$. Note that the ordering of the points in $\omega_{2}$ is preserved, e.g. $(r, 1)<(r, 2)$ and no two arguments are identical. The latter fact ensures the existence of the product $\prod_{r}$ whenever $\omega_{2}\left(x_{i}, x_{j}\right)$ are distributions. For the wave front set of $\omega_{n}$ one finds using Theorem 2

$$
\begin{aligned}
& \mathrm{WF}\left(\omega_{n}\right) \\
& =\bigcup_{\left(x_{1}, \ldots, x_{n}\right) \in M^{n}} \operatorname{WF}\left(\omega_{n}\left(x_{1}, \ldots, x_{n}\right)\right) \\
& \subseteq \bigcup_{\left(x_{1}, \ldots, x_{n}\right) \in M^{n}}\left(\bigcup_{P} \bigcup_{p} \bigoplus_{r_{p}}\left[M^{n-2} \times \mathrm{WF}\left(\omega_{2}\left(x_{\left(r_{p}, 1\right)}, x_{\left(r_{p}, 2\right)}\right)\right)\right]\right),
\end{aligned}
$$

where $p$ denotes a subset of $P$ and $r_{p}$ labels the elements of $p$.

Example 1. Consider the four-point distribution of a quasifree state:

$$
\begin{aligned}
& \omega_{4}\left(x_{1}, x_{2}, x_{3}, x_{4}\right)= \\
& \omega_{2}\left(x_{1}, x_{2}\right) \omega_{2}\left(x_{3}, x_{4}\right)+\omega_{2}\left(x_{1}, x_{3}\right) \omega_{2}\left(x_{2}, x_{4}\right)+\omega_{2}\left(x_{1}, x_{4}\right) \omega_{2}\left(x_{2}, x_{3}\right)
\end{aligned}
$$

We have

$$
P \in\left\{\left\{\left(x_{1}, x_{2}\right),\left(x_{3}, x_{4}\right)\right\},\left\{\left(x_{1}, x_{3}\right),\left(x_{2}, x_{4}\right)\right\},\left\{\left(x_{1}, x_{4}\right),\left(x_{2}, x_{3}\right)\right\}\right\}
$$

Let $P=\left\{\left(x_{1}, x_{3}\right),\left(x_{2}, x_{4}\right)\right\}$, then $p \in\left\{\left\{\left(x_{1}, x_{3}\right)\right\},\left\{\left(x_{2}, x_{4}\right)\right\},\left\{\left(x_{1}, x_{3}\right),\left(x_{2}, x_{4}\right)\right\}\right\}$. Assume $p=\left\{\left(x_{1}, x_{3}\right)\right\}$, then $r_{p}=\left(x_{1}, x_{3}\right)$, e.g. $x_{\left(r_{p}, 1\right)}=x_{1}$ and $x_{\left(r_{p}, 2\right)}=x_{3}$.

\footnotetext{
${ }^{2}$ I. g. we "close" up the light cone.
} 
Therefore

$$
\begin{aligned}
& \subseteq \bigcup_{\left(x_{1}, \ldots, x_{4}\right) \in M^{4}}\left(\left[M^{2} \times \mathrm{WF}\left(\omega_{2}\right)\right.\right. \\
& \left.\cup\left[\left(x_{1}, x_{2}\right)\right)\right] \cup\left[M^{2} \times \mathrm{WF}\left(\omega_{2}\left(x_{3}, x_{4}\right)\right)\right] \\
& \left.\left.\cup \operatorname{WF}\left(\omega_{2}\left(x_{1}, x_{2}\right)\right)\right) \oplus\left(M^{2} \times \mathrm{WF}\left(\omega_{2}\left(x_{3}, x_{4}\right)\right)\right)\right]
\end{aligned}
$$$$
\cup 2 \leftrightarrow 3
$$$$
\cup 2 \leftrightarrow 4)
$$$$
\equiv\left\{\left(x_{1}, k_{1} ; x_{2}, k_{2} ; x_{3}, 0 ; x_{4}, 0\right) \mid \quad\left(x_{1}, k_{1} ; x_{2}, k_{2}\right) \in \mathrm{WF}\left(\omega_{2}\right)\right\}
$$$$
\cup\left\{\left(x_{1}, 0 ; x_{2}, 0 ; x_{3}, k_{3} ; x_{4}, k_{4}\right) \mid \quad\left(x_{3}, k_{3} ; x_{4}, k_{4}\right) \in \mathrm{WF}\left(\omega_{2}\right)\right\}
$$$$
\cup\left\{\left(x_{1}, k_{1} ; x_{2}, k_{2} ; x_{3}, k_{3} ; x_{4}, k_{4}\right) \mid \quad\left(x_{1}, k_{1} ; x_{2}, k_{2}\right) \in \mathrm{WF}\left(\omega_{2}\right)\right. \text {; }
$$$$
\left.\left(x_{3}, k_{3} ; x_{4}, k_{4}\right) \in \mathrm{WF}\left(\omega_{2}\right)\right\}
$$

$\cup \cdots$

To prove the Corollary it is -by Theorem 2- necessary and sufficient to show that finite sums of $\mathrm{WF}\left(\omega_{n}\right)$ do not contain zero. We know the wave front sets of all distributions in Eqn. 12 explicitly (See Corollary 11). Consider the following two cases:

1. The direction in $\mathrm{WF}\left(\omega_{n}\right)$ associated to the first variable is not zero. Then its time component is strictly positive by Corollary 1. Since the time components of all directions associated to the first variable of $\mathrm{WF}\left(\omega_{n}\right)$ are always greater or equal to zero, the sum of all these directions can not vanish and the Corollary is proved.

2. The direction in $\mathrm{WF}\left(\omega_{n}\right)$ associated to the first variable is zero for all summands. Then all directions associated to the second variable must have time components greater or equal to zero and we apply the same argumentation as for the first variable. Note that it is excluded by the definition of the wave front set that all directions of all variables vanish simultaneously. Therefore there exists a variable $\left(x_{i}\right) i<n$ such that the analogon of (1) holds.

3.2. A local characterization of globally Hadamard states. One of the main motivations for using wave front sets in quantum field theory is the fact that they allow the specification of global properties locally. The following Theorem, which is one of the main results of Radzikowski's dissertation, gives a local characterization of globally Hadamard states.

Theorem 5 (Theorem 2.6 of $[12]$ ). Let $\omega_{2}$ be the two-point distribution arising from a state of a massive Klein-Gordon field propagating on a globally hyperbolic spacetime. If $\omega_{2}$ has wave front set as in Corollary $\mathbb{Z}$ above then $\omega_{2}$ is globally Hadamard.

Remark . Our assumption implies that $\omega_{2}$ fulfils the Klein-Gordon equation and has $i$ times the commutator distribution of $\square+m^{2}$ as its antisymmetric part. The Corollary 11 above may be viewed as the converse of this Theorem. 
The proof of this Theorem is rather long; we refer the reader to the dissertation of Radzikowski for the details. Radzikowski's Theorem 2.6 states the equivalence of the wave front set assumption and the globally Hadamard condition $\bmod C^{\infty}$. To prove it, he introduces the Feynman two-point distribution $\omega_{F}$ defined by $\omega_{F}:=i \omega_{2}+E^{+}$, where $E^{+}$is the advanced fundamental solution of $\square+m^{2}$. The assumption on the wave front set of $\omega_{2}$ uniquely fixes this distribution (up to $C^{\infty}$ ) to be the Feynman two-point distribution defined in [任. This in turn implies that $\omega_{2}$ is globally Hadamard $\bmod C^{\infty}$.

\section{The WAVE FRONT SET SPECTRUM CONDITION}

We have seen in the last section that the wave front set of a state can be used to characterize this state globally. However Equation (9) restricts the singular support of $\omega_{2}\left(x_{1}, x_{2}\right)$ to points $x_{1}$ and $x_{2}$ which are null related; hence $\omega_{2}$ is smooth for time-like or space-like related points. The latter smoothness is known to be true for reasonable quantum field theories on Minkowski space satisfying the true spectrum condition by the Bargmann-Hall-Wightman Theorem. For time-like related points however a similar general prediction on the smoothness does not exist. In order to include possible singularities at time like related points, Radzikowski extended in 112 the right hand side of Eqn. (9) to all causally related points; he proposed that the wave front set of the two-point distributions of any physical reasonable state should be contained in this extended set. He called this proposal the "wave front set spectrum condition' (WFSSC). He also proposed a WFSSC for higher n-point distributions and showed that both of his Definitions are compatible to the usual spectrum condition: Each $\omega_{n}$ fulfiling his WFSSC satisfies the true spectrum condition on Minkowski space $\bmod C^{\infty}$ (Theorem 4.10 of [12]) and vice versa. He gives further evidence on the legitimacy of his Definitions by linking them to the scaling limit condition of Fredenhagen and Haag [5]: Both Definitions imply the true spectrum condition in the scaling limit if this limit exists (Theorem 4.11 of 12). Unfortunately it can be shown that the n-point distributions for $n>2$ associated to a quasifree Hadamard state of a scalar field on a globally hyperbolic spacetime do not satisfy his WFSSC in general. Thus the WFSSC, at least for the higher n-point distributions, needs to be modified. We do not know a reasonable modification at the moment and therefore restrict ourself in this note to the WFSSC for the two-point distributions.

The counterexample mentioned above and the wish to include fields which are a composition of 'simple' fields (such as our field $K$ ), leads us to propose the following 'conic' WFSSQ ${ }^{3}$ for the two-point distributions, which is a slight modification of Radzikowski's original definition.

Definition 7 (WFSSC). The two-point distribution $\omega_{2} \in \mathcal{D}^{\prime}(M \times M)$ satisfies the wave front set spectrum condition (WFSSC) iff its wave front set $\operatorname{WF}\left(\omega_{2}\right)$ consists only of points $\left(x_{1}, k_{1}\right),\left(x_{2}, k_{2}\right) \in T^{*} M \backslash\{0\}$ such that $x_{1}$ and $x_{2}$ are causally related and $k_{1}$ is in the closed forward lightcone. Furthermore there are causal geodesics $\gamma_{i}$ joining $x_{1}$ and $x_{2}$ and vectors $k_{i}$ in the closed forward lightcone, such that $\sum_{i} k_{i}=k_{1}$ and the parallel transported vectors $k_{i}$ along $\gamma_{i}$ sum up to $-k_{2}$. I

\footnotetext{
${ }^{3}$ E. g. $\left(x, k_{1} ; y, l_{1}\right),\left(x, k_{2}, y, l_{2}\right) \in \mathrm{WF}\left(\omega_{2}\right) \Rightarrow\left(x, \lambda k_{1}+\mu k_{2} ; y, \lambda l_{1}+\mu l_{2}\right) \in \mathrm{WF}\left(\omega_{2}\right) \quad \forall \lambda, \mu>0$.

${ }^{4}$ Note that our definition allows the singularities to propagate along multiple curves $\gamma_{i}$ simultaneously.
} 


\section{Proof of Proposition 1}

It is first shown that $K$ is well defined and fulfils the following four Wightman axioms:

1. The distributional product of $A$ and $B, K$, is a well defined operator valued distribution on the GNS Hilbertspace $(H, \Omega, A, B)$ with domain

$$
D=\operatorname{Span}\left\{\Phi\left(f_{1}\right) \cdots \Phi\left(f_{n}\right)|\Omega>| \Phi \in\{A, B, K\}, f_{1}, \ldots, f_{n} \in C_{0}^{\infty}(M)\right\}
$$

2. $D$ is dense in $H$.

3. For all $f \in C_{0}^{\infty}(M), K(f)$ leaves $D$ invariant.

4. For all $f \in C_{0}^{\infty}(M), K(f)$ is local (e.g. bosonic).

We finish the proof showing that the two-point distribution of $K$ with respect to the "vacuum" $\Omega$ fulfils the WFSSC (Definition 7).

1. Let $D^{\prime}=\operatorname{Span}\left\{\Psi=K\left(f_{1}\right) \cdots K\left(f_{n}\right)|\Omega>| f_{i} \in C_{0}^{\infty}(M)\right\}$. We remark that the definition of $D^{\prime}$ is only formal at this stage. To show that $K$ is well defined on $D^{\prime}$, it is sufficient to show that

$$
\|K(f) \Psi\|<\infty \quad \forall f \in C_{0}^{\infty}(M), \quad \Psi \in D^{\prime}
$$

Inserting the definitions of $K$ and $\Psi$ in Equation (13) we obtain in local coordinates:

$$
\begin{aligned}
&\|K(f) \Psi\|^{2}=<K(f) K\left(f_{1}\right) \cdots K\left(f_{n}\right) \Omega \mid K(f) K\left(f_{1}\right) \cdots K\left(f_{n}\right) \Omega> \\
&=<\Omega \mid K\left(\bar{f}_{n}\right) \cdots K\left(\bar{f}_{1}\right) K(\bar{f}) K(f) K\left(f_{1}\right) \cdots K\left(f_{n}\right) \Omega> \\
&= \int \cdots \int d \mu_{x_{n}} \cdots d \mu_{x_{1}} d \mu_{x} d \mu_{y} d \mu_{y_{1}} \cdots d \mu_{y_{n}} \\
&{ }_{A} \omega_{2 n+2}\left(x_{n}, \ldots, x_{1}, x, y, y_{1}, \ldots, y_{n}\right) \cdot \\
&{ }^{B} \omega_{2 n+2}\left(x_{n}, \ldots, x_{1}, x, y, y_{1}, \ldots, y_{n}\right) \\
& \bar{f}_{n}\left(x_{n}\right) \cdots \bar{f}_{1}\left(x_{1}\right) \bar{f}(x) f(y) f_{1}\left(y_{1}\right) \cdots f_{n}\left(y_{n}\right)
\end{aligned}
$$

Equation (14) separates into the distributional product of two $2 n+2$-point distributions since we are dealing with a product state. $\omega_{2 n+2}$ denotes the $2 n+2$-point distribution of the corresponding basic field $A$ or $B$ and the r.h.s. of Eqn. (14) defines $\|K(f) \Psi\|^{2}$. All $2 n+2$-point distributions arise from quasifree states. Hence they decay into the sum of products of two-point distributions. However using Corollary 2 one sees that arbitrary (finite) products of these distributions with each other exist and are again distributions. This ensures the finiteness of Eqn. (14). Now suppose we replace one or more $K\left(f_{i}\right)$ in Eqn. (14) by $\phi\left(g_{1}, \ldots, g_{k}, \tilde{g}_{1}, \ldots, \tilde{g}_{l}\right) \equiv A\left(g_{1}\right) \cdots A\left(g_{k}\right) B\left(\tilde{g}_{1}\right) \cdots B\left(\tilde{g}_{l}\right)$ with $g_{1}, \ldots, \tilde{g}_{l} \in C_{0}^{\infty}(M)$. Redoing the calculation in Eqn. (14) one ends up again with various products of two-point distributions. However all two-point distributions which include parts of the " $\phi$ 's" are in fact smooth functions (for example $\left.{ }^{A} \omega_{2}\left(g_{i}, x\right) \in C^{\infty}(M) ;{ }^{A} \omega_{2}\left(g_{i}, g_{j}\right) \in \mathbb{C}\right)$. Thus replacing an operator $K\left(f_{i}\right)$ by $\phi\left(g_{1}, \ldots, \tilde{g}_{l}\right)$ does not change the finiteness of the whole equation. Furthermore, since the products in Eqn. (14) are again distributions, it follows that $\tilde{K}_{\Psi \Psi^{\prime}}: C_{0}^{\infty}(M) \ni f \mapsto<\Psi \mid K(f) \Psi^{\prime}>$ is well defined and continuous for all $\Psi, \Psi^{\prime} \in D$. This proves the assertion.

2. is obvious since $H$ is the GNS Hilbertspace of the basic fields, e.g. the "vacuum" $\Omega$ is cyclic for $A$ and $B$. 
3. follows directly from our definition of $D$.

4. To prove 4 . one notes that $A$ and $B$ mutually commute and

$$
[A(f), A(g)]_{-}=[B(f), B(g)]_{-}=0 \quad \text { if } \operatorname{supp}(f) \subset \operatorname{supp}(g)^{c},
$$

for causally disjoint arguments. $\operatorname{supp}(g)^{c}$ denotes the causal complement of the support of $g$. Therefore

$$
[K(f), K(g)]_{-}=0 \quad \text { if } \operatorname{supp}(f) \subset \operatorname{supp}(g)^{c},
$$

Remark . $\quad-D^{\prime}$ is not dense in $H$ since for example $\Phi=A(f) \mid \Omega>$ can not be approximated by elements in $D^{\prime}: \forall D^{\prime} \ni \Psi$ we have $\|\Phi-\Psi\|^{2}=\|\Phi\|^{2}+\|\Psi\|^{2}$ since $\langle\Phi, \Psi\rangle=0$. The latter equation is valid, since $\langle\Phi, \Psi\rangle$ decays into a product of n-point distributions, one of which has an even, the other an odd number of arguments; such combinations always vanish.

- The vector $\Omega$ is not cyclic for the field $K$.

To show that the two-point distribution of $K$ in $\omega$ fulfils the WFSSC, we calculate its wave front set. In local coordinates, we obtain:

$$
\begin{aligned}
{ }^{K} \omega_{2}(f, g) & :=<\Omega \mid K(f) K(g) \Omega> \\
& =\iint_{M \times M} d \mu_{x} d \mu_{y}{ }^{A} \omega_{2}(x, y)^{B} \omega_{2}(x, y) f(x) g(y)
\end{aligned}
$$

Equation (15) is just the product of distributions on the manifold $M$ and by Corollary 1 above

$$
\begin{aligned}
& \mathrm{WF}\left({ }^{A} \omega_{2}\right)=\mathrm{WF}\left({ }^{B} \omega_{2}\right)=\left\{(x, k),\left(y, k^{\prime}\right) \in\left(T^{*} M \times T^{*} M\right) \backslash\{0\} \mid\right. \\
&(x, k)\left.\sim\left(y,-k^{\prime}\right), k^{0}>0\right\} .
\end{aligned}
$$

Since $\operatorname{WF}\left({ }^{A} \omega_{2}\right) \oplus \operatorname{WF}\left({ }^{A} \omega_{2}\right)$ fulfils the assumption of Theorem 2, we deduce that the kernel of (15) defines a unique distribution, namely ${ }^{K} \omega_{2}$ with wave front set contained in

$$
W:=\left(\mathrm{WF}\left({ }^{A} \omega_{2}\right) \oplus \mathrm{WF}\left({ }^{A} \omega_{2}\right)\right) \cup \mathrm{WF}\left({ }^{A} \omega_{2}\right)
$$

We finish the proof showing that the elements of $W$ fulfil the assumption of Definition 07 above:

Consider an element $(x, k ; y, l)$ of $W$. If it is contained in $\operatorname{WF}\left({ }^{A} \omega_{2}\right)$, everything is proved, since $\mathrm{WF}\left({ }^{A} \omega_{2}\right)$ is assumed to be the two-point distribution of a Hadamard state. On the other hand if this element is contained in $\operatorname{WF}\left({ }^{A} \omega_{2}\right) \oplus \operatorname{WF}\left({ }^{A} \omega_{2}\right)$, then there exist covectors $k_{1}, k_{2}$ and $l_{1}, l_{2}$ respectively with $k_{1}+k_{2}=k$ and $l_{1}+l_{2}=l$, such that $\left(x, k_{i} ; y, l_{i}\right) \in \mathrm{WF}\left({ }^{A} \omega_{2}\right)$ for $i=1,2$. With the corresponding curves $\gamma_{i}$ we have

1. $k_{1}+k_{2} \in V_{+x}$ since $k_{i} \in V_{+x}$ by assumption.

2. $g_{12}^{\gamma_{1}} k_{1}+g_{12}^{\gamma_{2}} k_{2}=-\left(l_{1}+l_{2}\right)$ since $\left(x, k_{i}\right) \sim\left(y,-l_{i}\right)$

$V_{+x}$ is the closed forward lightcone at $x \in M$ and $g_{12}^{\gamma_{i}}$ denotes the parallel transport along the curve $\gamma_{i}$ from $x_{1}$ to $x_{2}$.

Remark . - In general there exists more than one causal curve connecting $x_{1}$ and $x_{2}$. The modification in Definition 7 which allows multiple curves $\gamma$ to contribute is necessary because in the generic case the parallel transport is path dependent, e.g.

$$
g_{12}^{\gamma_{1}} k_{1}+g_{12}^{\gamma_{1}} k_{1} \neq-\left(l_{1}+l_{2}\right) \neq g_{12}^{\gamma_{2}} k_{1}+g_{12}^{\gamma_{2}} k_{1}
$$


- The two-point distribution of our state is smooth for time-like related points. It is therefore possible to strengthen Definition 0 by demanding that the singular support of $\omega_{2}$ should contain light-like related points only.

\section{Summary and Outlook}

In this note it has been proved that the distributional product of two scalar fields on a globally hyperbolic spacetime gives a new Wightman field on this manifold. Obviously the result extends to all cases where the definition of a quasifree Hadamard state makes sense. The free Dirac field is an example, which will be used in a forthcoming paper to calculate the renormalized stress energy tensor of an analogon of the free Wess-Zumino model on a manifold. Moreover it has been shown that this Wightman field satisfies the new wave front set spectrum condition (WFSSC) on a manifold. In the opinion of the author this condition will turn out to be a valuable tool for quantum field theory on curved spacetimes. It should for example enable some kind of perturbation theory on manifolds in the future. But even on Minkowski spacetime the study of wave front sets of more realistic models (for example QED) might result in a deeper understanding of the Wightman axioms and the true spectrum condition.

An extension of the WFSSC to higher $n$-point distributions remains to be found, since the proposal of Radzikowski is not acceptable.

Acknowledgement . The author has benefited from discussions with many members of the II. Institut für Theoretische Physik at the University of Hamburg. In particular he wishes to thank Prof. Fredenhagen for his guidance during this research, for many stimulating discussions and for valuable comments on the manuscript. Special thanks are due to $\mathrm{R}$. Verch for helpful hints and a careful reading of the manuscript. M. Radzikowski is gratefully acknowledged for having made the results of his $\mathrm{PhD}$. thesis available prior to publication.

\section{REFERENCES}

1. H. J. Borchers, On the structure of the algebra of the field operators, Nuovo Cimento 24 (1962), 214.

2. M. A. Castagnino and D. D. Harari, Hadamard renormalization in curved space-time, Ann. of Phys. 152 (1984), 85-104.

3. B. S. DeWitt and R. W. Brehme, Radiation damping in a gravitational field, Ann. Phys. 9 (1960), 220-259.

4. J. J. Duistermaat and L. Hörmander, Fourier integral operators II., Acta Math. 128 (1972), 183.

5. K. Fredenhagen and R. Haag, Generally covariant quantum field theory and scaling limit, Comm. Math. Phys. 108 (1987), 91.

6. S. A. Fulling, Aspects of quantum field theory in curved space-time, Cambridge University Press, Cambridge, 1989.

7. S. A. Fulling, F. J. Narcowich, and R. M. Wald, Singularity structure of the two-point function in quantum field theory in curved spacetime, II, Ann. Phys. (NY) 136 (1981), 243-272.

8. R. Haag and D. Kastler, An algebraic approach to quantum field theory, J. Math. Phys. 5 (1964), 848.

9. R. Haag, H. Narnhofer, and U. Stein, On quantum field theory in gravitational background, Comm. Math. Phys. 94 (1984), 219-238.

10. L. Hörmander, Fourier integral operators I., Acta Math. 127 (1971), 79.

11. B. S. Kay and R. M. Wald, Theorems on the uniqueness and thermal properties of stationary, nonsingular, quasifree states on spacetimes with a bifurcate Killing horizon, Phys. Rep. 207 (1991), no. 2, 49-136. 
12. M. J. Radzikowski, The Hadamard condition and Kay's conjecture in (axiomatic) quantum field theory on curved space-time, Ph.D. thesis, Princeton University, October 1992.

13. M. Reed and B. Simon, Methods of modern mathematical physics II, Academic Press Inc. London, 1975.

14. M. E. Taylor, Pseudodifferential operators, Princeton University Press, Princeton, New Jersey, 1981.

15. A. Uhlmann, Über die Definition der Quantenfelder nach Wightman und Haag, Wiss. Zeitschrift Karl Marx Univ. 11 (1962), 213.

16. R. Verch, Nuclearity, split property, and duality for the Klein-Gordon field in curved spacetime, Lett. Math. Phys. 29 (1993), 297-310.

17. L Local definiteness, primarity and quasiequivalence of quasifree Hadamard quantum states in curved spacetime, Comm. Math. Phys 160 (1994), 507-536. 